\title{
A droplet deformation and fragmentation in a high speed air jet
}

\author{
Maryam Medghalchi, Nasser Ashgriz \\ Department of Mechanical and Industrial Engineering \\ University of Toronto \\ Toronto, Canada \\ medghalchi@mie.utoronto.ca, ashgriz@mie.utoronto.ca
}

\begin{abstract}
Impingement of a gas jet on a suspended water droplet is modeled numerically. The jet velocity and jet diameter effects on the penetration length and the shape of the gas tunnel inside the droplet are studied. The gas tunnel inside the droplet is smaller on the windward and larger on the leeward of the droplet. Different types of breakup processes are identified and categorized according to jet to droplet diameter ratio, as well as the droplet Weber number.
\end{abstract}

\section{Keywords; droplet breakup; fragmentation; jet ;}

\section{INTRODUCTION}

The droplet deformation and fragmentation are involved in many different systems, such as in fuel injection systems and powder production system. The process of droplet fragmentation is a complex one involving large interface deformations and interface breakups, and mostly in turbulent conditions. In order to characterize this complex process, usually the droplet breakup process is studied in a more controlled condition. For instance, a single droplet is injected into a high velocity gas flow and its deformation and breakup is determined at different gas flow velocities. Almost all prior studies on the droplet deformation and breakup have considered a droplet in a gaseous crossflow, where the gaseous crossflow is much larger than the droplet diameter. The present study has taken a different approach. Here, the deformation and a breakup of a droplet subject to a cross flow that its diameter is in the order of the droplet diameter is considered.

The droplet behavior is generally characterized using a Reynolds, a Weber and an Ohnesorge number defined as [1]:

$$
\begin{gathered}
R e=\frac{\rho_{l} U D_{\text {drop }}}{\mu_{l}} \\
W e=\frac{\rho_{l} U^{2} D_{\text {drop }}}{\sigma} \\
O h=\frac{\sqrt{W e}}{R e}=\frac{\mu_{l}}{\sqrt{\rho_{l} D_{\text {drop }} \sigma}}
\end{gathered}
$$

where $D_{\text {drop }}$ is the droplet diameter, $U$ is the relative velocity between the gas and the droplet, $\rho_{l}$ is the liquid density, $\mu_{l}$ is the liquid viscosity, and $\sigma$ is the coefficient of surface tension.
Gelfand [2] categorized the droplet breakup into several different modes as a function of $W e$ and $O h$. The following modes are identified: Vibrational, bag breakup, multimode, sheet thinning and catastrophic modes. In the vibrational mode, the drop oscillates until it divides into smaller droplets. In the bag breakup mode, a thin shell is generated, which later bursts forming many small fragments. The multimode breakup is similar to the bag breakup except that it includes a stamen, which forms in the leeside of the droplet (opposite to the gaseous flow). In the sheet thinning breakup, the surface of the droplet is sheered forming a thin liquid sheet, which later breaks forming small fragments. In the catastrophic breakup, the droplet instantly fragments into many small droplets. Guildenbacher et al. [3] reviewed the droplet secondary breakup and they found that viscous forces do not play a significant role at moderate Weber numbers. There are many other studies on the droplet breakup, and readers are referred to see the following references [4-10].

In the present study, we consider the impingement of a gas jet on a droplet, where the gas jet diameter is about the size of the droplet.

\section{Methodology}

Figure 1 shows the problem parameters. An initially stationary droplet with diameter $D_{\text {drop }}$ is exposed to a gas jet with diameter and velocity. The dynamics of the droplet deformation and breakup is simulated numerically.

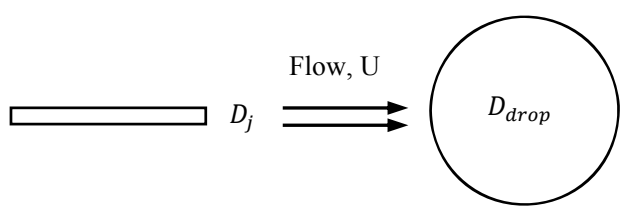

Figure 1: Schematic diagram of the nozzle and droplet

The numerical code used is ANSYS/FLUENT. The gasliquid interface is tracked by Volume Of Fluid (VOF) method. Continuity, momentum and energy conservation equations are solved with presence of the gravity. The turbulence model is RANS, standard $k-\varepsilon$ [11]. 
The governing equation are:

$$
\begin{gathered}
\frac{1}{\rho_{q}}\left[\frac{\partial}{\partial t}\left(\alpha_{q} \rho_{q}\right)+\nabla \cdot\left(\alpha_{q} \rho_{q} \vec{u}_{q}\right)=0\right] \\
\frac{\partial}{\partial t}(\rho \vec{u})+\nabla \cdot(\rho \vec{u} \vec{u})=-\nabla p+\nabla \cdot\left[\mu\left(\nabla \vec{u}+\nabla \vec{u}^{T}\right)\right]+\rho g+\vec{F} \\
\frac{\partial}{\partial t}(\rho E)+\nabla \cdot(\vec{u}(\rho E+p))=\nabla \cdot\left(k_{e f f} \nabla T\right)
\end{gathered}
$$

where in equation (4), $\alpha_{q}$ is the volume fraction of the secondary phase, $\rho_{q}$ is the density of the secondary phase and $u$ represents the velocity. In equation (6), $k_{e f f}$ is the effective thermal conductivity.

The continuous phase volume fraction is determined by, $\sum_{q=1}^{n} \alpha_{q}=1$ and $\rho=\sum \alpha_{q} \rho_{q}$, and $\mu=\sum \alpha_{q} \mu_{q}$. In equation (6), $E$ is the mass average of specific heats of phases.

$$
E=\frac{\sum_{q=1}^{n} \alpha_{q} \rho_{q} E_{q}}{\sum_{q=1}^{n} \alpha_{q} \rho_{q}}
$$

\section{A. Numerical Setup}

To reduce the computational time the numerical simulations are run on a $3 \mathrm{~cm} \times 1 \mathrm{~cm}$ rectangular 2D-axisymmetric geometry with the total of $1,140,000$ computational cells in fine mesh cases. In coarse mesh cases, the number of cells is reduced to 58,000 .

The air density is determined using the ideal gas equation of state and the droplet density is assumed to be constant.

Three different nozzle sizes with different jet velocities and droplet sizes are simulated. Results are presented in the following section.

\section{RESULTS AND DISCUSSION}

Two different droplet sizes of $2.4 \mathrm{~mm}$ and $4.8 \mathrm{~mm}$, and three different air jet diameters of $0.2 \mathrm{~mm}, 0.4 \mathrm{~mm}$ and $3.8 \mathrm{~mm}$ with two different gas velocities of $49.7 \mathrm{~m} / \mathrm{s}$ and $99.4 \mathrm{~m} / \mathrm{s}$ are considered.

Figure 2 shows the impingement of a $0.2 \mathrm{~mm}$ air jet with jet velocity of $49.7 \mathrm{~m} / \mathrm{s}$ on a $2.4 \mathrm{~mm}$ water droplet. The Weber number based on droplet diameter for this case is $W e_{\text {Drop }}=124$. The air jet impinges on the droplet and penetrates into the droplet forming an air tunnel. The liquid surface tension forces resist the jet flow. Therefore, the incoming gas goes through a circulation pattern forming a large cavity inside the droplet (see the evolution of the cavity in Figure $2 \mathrm{a}$ to $2 \mathrm{~d}$ ). In addition, the liquid at the windward of the droplet is pushed together to close the jet opening zone. Moreover, Figure 2 shows that droplet oscillates between circular and oval shape.

Figure 3 shows the droplet deformation for a gas jet velocity of $99.4 \mathrm{~m} / \mathrm{s}$ (Weber number 496) for the same jet and droplet diameters as in Figure 2. In this case, the air jet is strong enough to overcome droplet inertia and surface tension forces, and pierces through the droplet.
Figure 4 shows the velocity vectors on the droplet plane for a jet diameter of $0.4 \mathrm{~mm}$ and a droplet diameter of $4.8 \mathrm{~mm}$. Both the jet diameter and droplet diameter are increase such that their ratio is kept the same as those shown in Figure 2 and Figure 3. The jet velocity in this case is $49.7 \mathrm{~m} / \mathrm{s}$ (same as Figure 2, however, the Weber number is 248). In Figure 4 , the jet impinges, penetrates and pierces through the droplet. A similar type of cavity as in Figure 2 is observed in this case. However, in this case, because of higher inertia, the jet can force through the droplet. The bursting of a liquid layer that is formed at the time that the gas jet emerges out of the droplet results in the formation of several small droplets.
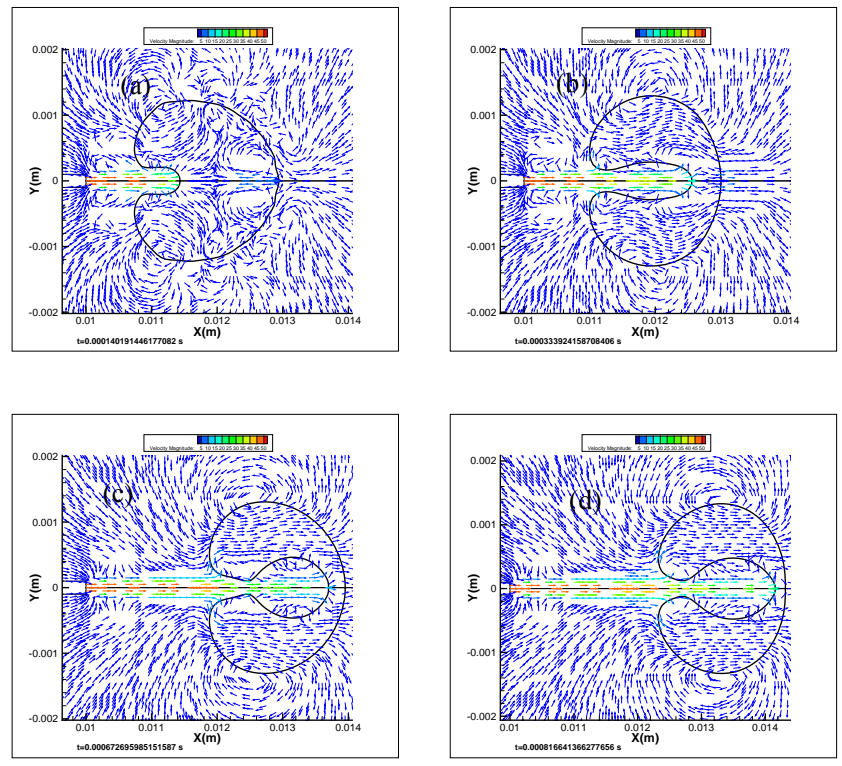

Figure 2: Velocity vectors and droplet for the jet diameter of $0.2 \mathrm{~mm}, U=49.7 \mathrm{~m} / \mathrm{s}$ and $W e_{\text {Drop }}=124$
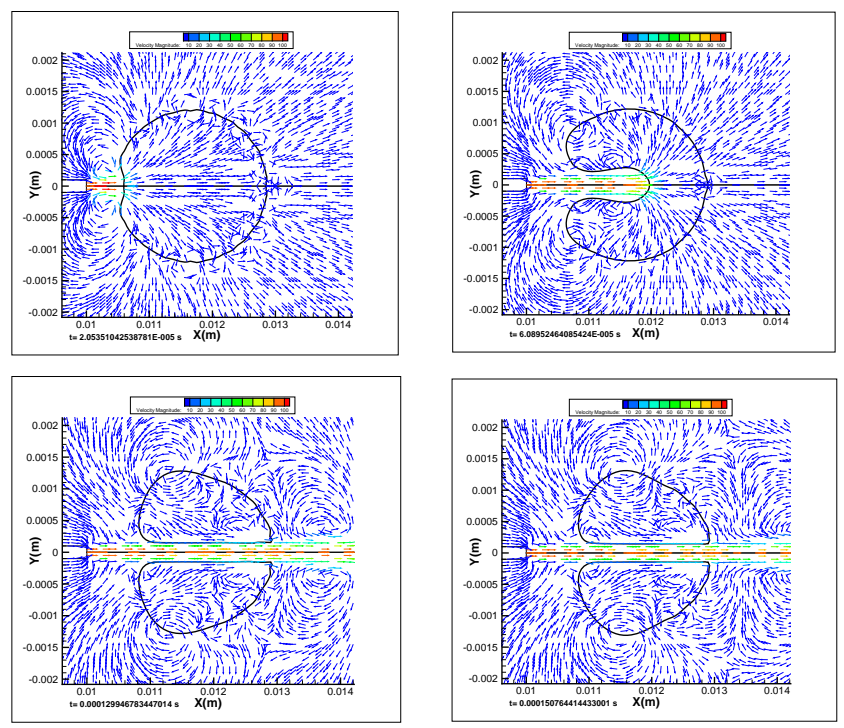

Figure 3: Velocity vectors and droplet for the jet diameter of $0.2 \mathrm{~mm}, U=99.4 \mathrm{~m} / \mathrm{s}$ and $W e_{\text {Drop }}=496$ 

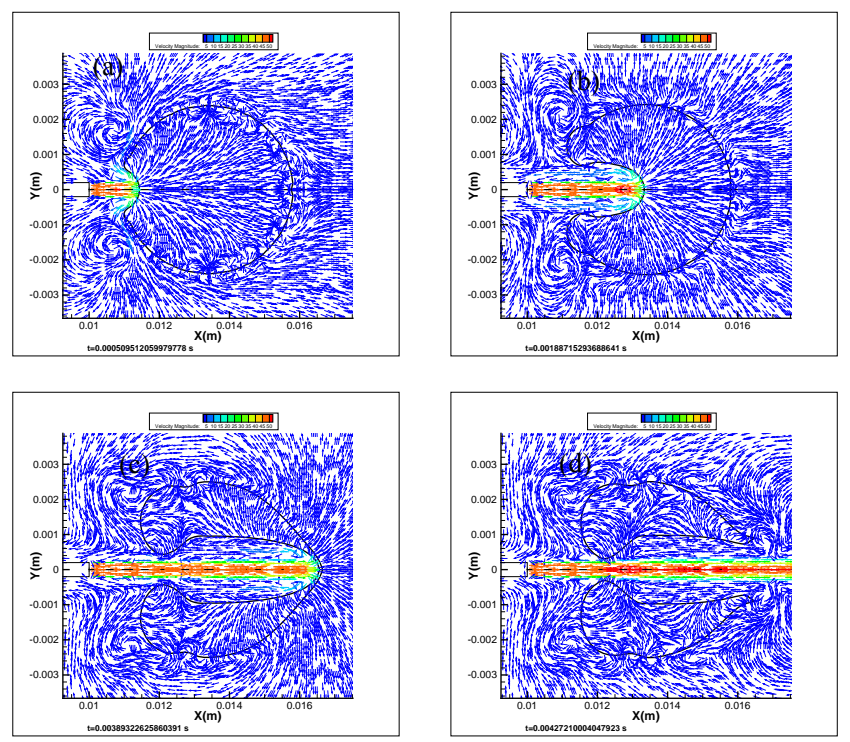

Figure 4: Velocity vectors and droplet for the jet diameter of $0.4 \mathrm{~mm}$, droplet diameter of $4.8 \mathrm{~mm}, U=49.7 \mathrm{~m} / \mathrm{s}$ and $W e_{\text {Drop }}=248$

Figure 5 shows droplet breakup for the same jet as in Figure 4 but with a smaller droplet size $(2.4 \mathrm{~mm})$. The jet diameter to droplet diameter ratio and the Weber number are reduced by $50 \%$. Comparing two figures shows that the larger droplet breaks earlier than the smaller droplet. Moreover the shape of the air tunnel inside the droplet is different in two cases. For the larger droplet (Figure 4) the air tunnel is long and thin, however, in the smaller droplet, the air tunnel is wide and short. Furthermore, the droplet in Figure 5, is significantly deformed, indicating that the inner flow behavior influences the droplet shape.

Comparing Figure 2 and Figure 5, which have the same droplet size and different gas jet sizes, shows that by doubling the jet size and keeping the Weber number constant the droplet stretched by the flow and its thickness is decreased until it bursts.

Figure 6 shows the droplet breakup for the jet diameter of $D_{j e t}=3.8 \mathrm{~mm}$ and the droplet size of $D_{\text {Drop }}=2.4 \mathrm{~mm}$. Air jet flattens the windward of the droplet and does not pierce the droplet. The droplet deforms from circular shape to a thin disk and the fragments are released from the top and bottom of the droplet.
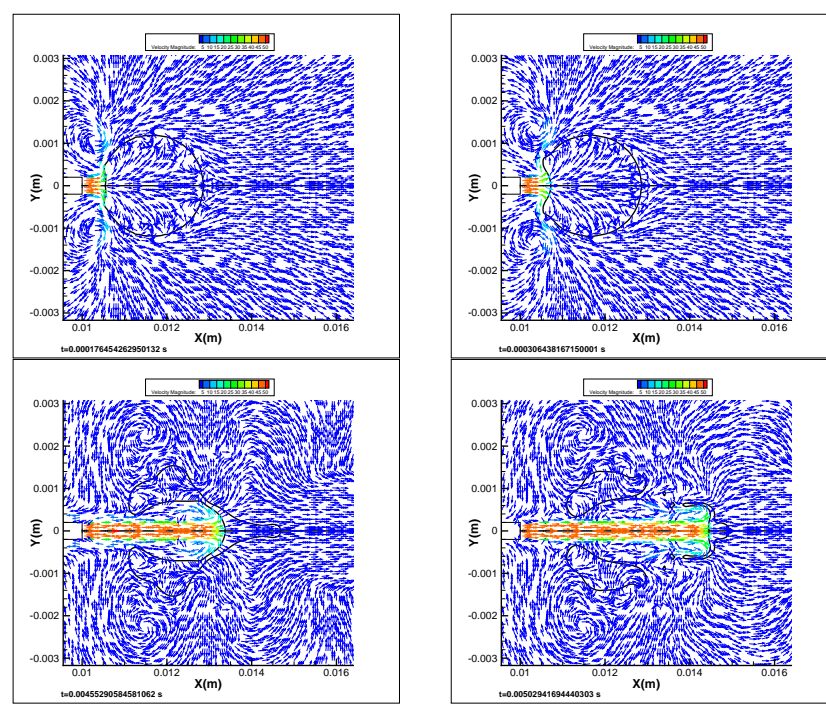

Figure 5: Velocity vectors and droplet for the jet diameter of $D_{\text {jet }}=0.4 \mathrm{~mm}$, droplet diameter of $D_{\text {drop }}=2.4 \mathrm{~mm}, U=$ $49.7 \mathrm{~m} / \mathrm{s}$ and $W e_{\text {Drop }}=124$
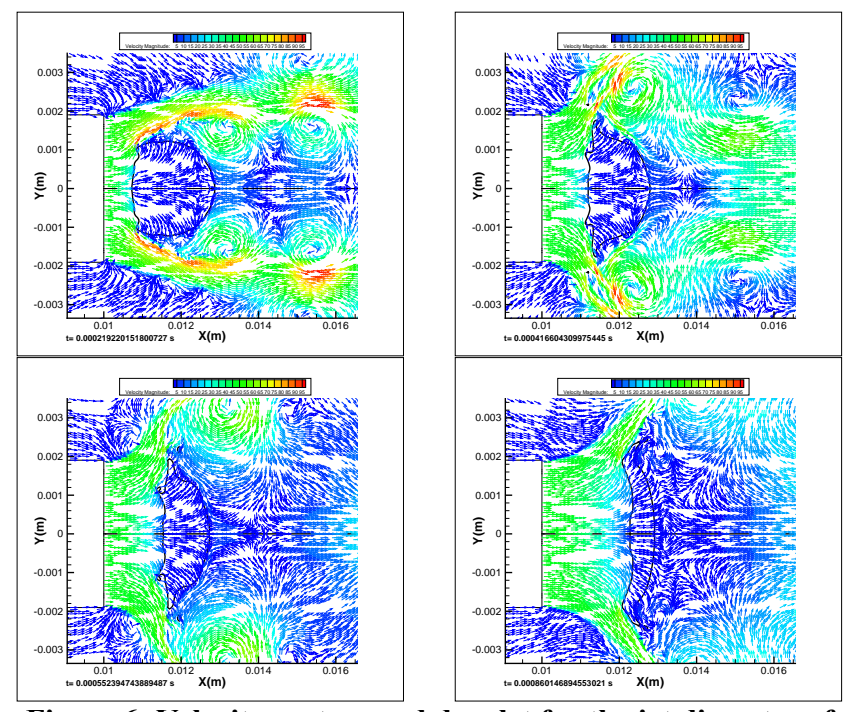

Figure 6: Velocity vectors and droplet for the jet diameter of $3.8 \mathrm{~mm}, U=49.7 \mathrm{~m} / \mathrm{s}$ and $W e_{\text {Drop }}=124$

\section{CONCLUSION}

Impingement of a gas jet of a liquid droplet for cases with gas jet diameter being smaller or in the order of droplet diameter are simulated numerically. The results indicate that the droplet deformation behavior is quite different than those reported in the literature for droplet deformation in a gaseous cross flow. The results show that for the jet to droplet diameter ratios less than one, a gas tunnel is formed inside the droplet that may have different configurations. At low gas jet velocities, a closed tunnel is formed which may result in an air entrapment inside the droplet. At larger gas velocities, the jet may penetrate through the droplet with less of an effect on the droplet deformation. 


\section{REFERENCES}

[1] G. Strotos , I. Malgarinos, N. Nikolopoulos, M. Gavaises, "Predicting droplet deformation and breakup for moderate Weber numbers," Int. Journal of Multiphase Flow, vol. 85, pp. 96-109, 2016.

[2] B. E. Gelfand, "Droplet breakup phenomena in flow with velocity lag," Prog. Energy Combust. Sci. Vol. 22, pp. 201-265, 1996.

[3] D. R. Guildenbecher, C. Lo'pez-Rivera, P. E. Sojka, "Secondary atomization," Exp Fluids, Vol. 46, pp. 371-402, 2009.

[4] G. M. Faeth, L. P. Hsiang and P. K. Wu "Structure and breakup properties of sparayes", Int. Journal of Multiphase Flow, vol. 21, pp. 99-127, 1995.

[5] A. Ashgriz, "Handbook of atomization and sprays," Springer, 2011.

[6] M. Pilch and C. A. Erdman, "Use of breakup time data and velocity history data to predict the maximum size of stable fragments for acceleration-included breakup of a liquid cdrop," Int. J. Multiphase Flow Vol. 13, No. 6, pp. 741-757. 1987.

[7] A. Mashayekh and N.Ashgriz, "Model for deformation of drops and liquid jets in gaseous crossflows," AIAA journal, Vol. 47, No. 2, pp. 303-313, 2009.

[8] T. Kékesi, G. Amberg, L. Prahl Wittberg, "Drop deformation and breakup,” Int. J. Multiphase Flow Vol. 66, pp. 1-10, 2014.

[9] S. A. Krzeczkowski, "Measurment of liquid droplet disintegration mechanisms, ” Int. J. Maltiphase Flow Vol. 6, pp. 227-239, 1979.

[10] Z. Liu and R. D. Reitz, " An Analysis of the distortion and breakup mechanisms of high speed liquid drops, ” Int. J. Multiphase Flow Vol. 23, No. 4, pp. 631-650, 1997.

[11] Ansys/Fluent theory guide 2017. 